\title{
Investigation and Optimization of Scheduling System in Sohar University using Genetic Algorithm (GA)
}

\author{
Maryam Alwashahi \\ Sultanate of Oman, Sohar University
}

\begin{abstract}
This paper presents the results of an investigation, and optimization of scheduling system in Sohar University (as a case study) using the genetic algorithm (GA). GA techniques are useful for solving real-world scheduling problem such as timetable which is a complex work and usually done manually. This work focuses on scheduling courses timetable to allocate events (time, subject, and lecturer) in an appropriate way by using the available resource and assists to avoid conflicts. The algorithms explored different operator of GA such as crossover, mutation, and selection mechanism that's applied to set of chromosomes. The testing has been produced using different parameters of population size, crossover, and mutation probability. Two point crossovers implemented to the timetable to obtain the optimal solution using various probabilities of crossover. The result shows the rate of crossover and mutation equal to 100 performed best optimal solutions. This paper recommended to enhance the fitness function and used different selection mechanism to the algorithm.
\end{abstract}

\section{General Terms}

Genetic Algorithm, Scheduling timetable, crossover, mutation

\section{Keywords}

Timetable problems, Genetic Algorithm (GA), Nondeterministic Polynomial (NP)

\section{INTRODUCTION}

The computers of fifth generations are dealing with Artificial Intelligence techniques to the optimization solution in decision-making and many more purposes. The GA is one of artificial intelligence and modern methods that resolve complex issues such as scheduling time tables. GA was developed by John Holland in 1975. The GA can be used to find solution for complex issues, being large and having the largest number of alternative solutions, involved in many areas to give an initial solution of the matter or to improve the solution for the problems that have been already existed. GA can be used in many areas including Machine Learning, Image Processing, Neural Network and Optimization.

The most common problem in educational institute is the timetable problem. It is considered as Non-deterministic Polynomial (NP) problem. There are a different number of timetable problems in the literature based on the type of institute. There are three main classifications of timetable presented by Schafer [1] as follows:

1. Subject timetable: It is concerned with the scheduling for the lecturer per semester at the university. It considers about minimizing the confliction of lecturer classes and common student group. That means, the student group cannot have more than one subject, and no lecturer is allocated to more than one class at the same time.

2. School timetable: It is concerned with the weekly class scheduling and avoids any teacher to meet more than one class at the same time.

3. Examination timetable: It is concerned with the scheduling of the exams for the university subjects and avoids overlapping of subjects.

Sohar University uses manual way to design timetable then enters it into the $\mathrm{E}$ - register system since, this manual process is frustrating and time consuming, and there was a requirement to apply modern computing techniques to automatically generate the timetable. In this paper, Genetic Algorithm has been used for the investigation and optimization of timetable scheduling system in Sohar University.

\section{BACKGROUND}

The timetable scheduling problem is common to all educational institutions. The main requirement is to eliminate or at least minimize the number of conflicts in the timetable. Sohar University is also having the same problem to schedule teachers, students, and classes to fully utilize all available resources. It is a regular practice for administrators to prepare and design timetable before students start their new semester. Class timetable was done manually, where the schedule has been generated one month earlier before next semester start. The class timetabling should focus on the aspects of place, time, subject, time-slot, and teacher. For most of the scheduling problems it has been shown that they are NP-hard, and that they cannot be solved in polynomial time using a deterministic algorithm [2]. GA is a nature inspired search and optimization technique. It works on the set of population in the form of chromosomes. In the timetable, certain factors called hard constraints should be considered like working day hours, time slot, class, subject, and student group and teacher availability. The hard constraints should be considered when it comes to design a timetable in a way that the overlapping between classes, teacher or student group can be avoided. Thus, the GA will assist the educational institutes to generate a timetable in less time, with more accuracy and efficiency [3].

The objectives of this paper are the given below:

1. To propose a suitable methodology and efficient algorithm in solving scheduling problem.

2. To develop a framework of the GA in a timetable system of Sohar University as Case Study.

3. To evaluate the performance of the proposed system. 


\section{RELATED WORK}

Timetable problem is a case of schedule problem. Scheduling timetable contains of teachers, numbers of students, and classroom into a fixed set of periods. An optimal schedule would be where no students, teacher or classroom is used more than once at any given time. The problem of the timetable can describe as finding a schedule where the classroom, teachers, and student's combination within the same period have a minimal number of overlapping elements. Therefore, automatic generation of timetables is an essential requirement. A good survey on automatic timetabling is given in [1]. A good literature survey on timetabling techniques is given by Sandhu [4] in his thesis. He discussed various techniques and algorithms used in timetabling generations.

GA is one of the most popular optimal solutions for scheduling [5, 6]. GA is a heuristic algorithm based on natural selection, and evolution [7]. The GA is a nature inspired search and optimization techniques. It works with a set of solutions called population, which are represented by chromosomes. The solutions from one population are taken to new and better population. The selection of parents is taken in the form of new solutions (offspring), which is based on their fitness value. The better fitness of a parent, the more chances they can survive and reproduce. This process is repeated until a feasible solution is found or it meets termination criteria [8]. The detailed concepts and applications of GA can be found in [9].

Ehsan, Zahra, and Mohammad [10] examined the course scheduling in the Islamic Azad University of Iran as real system. They used hybrid algorithm for satisfying all the constraint of generating the timetable automatically. Chinnasri et al. [11] compares and evaluated between the operators of Partially Matched crossover (PMX), Cycle crossover (CX), and Order crossover (OX) using GA for University Course Timetabling Problem (UCTP). They investigate real UCTP at Rangsit University, Thailand, to identify the different probability of mutation, crossover with different number of generations. In their case study, they got various results, which show that OX works more effective than others for generating realistic timetable for the course and CX is a good operator in finding feasible and optimal timetable.

Lukas et al, [12] proposed a method for solving this time table problem by using genetic algorithm combined with heuristic search. The role of genetic algorithm is to determine the sequence of all courses to be scheduled in one group, whereas the role of heuristic search is to determine time slots used to schedule the courses. They applied their approach for timetabling in university of Peliata Harapan.

\section{METHODOLOGY AND ALGORITHM}

The development is classified into five phases. The flows of the phases are problem identification, theory building, system development, experimentation and discussion and evaluation. The figure 1 illustrates the methodology used in this paper:

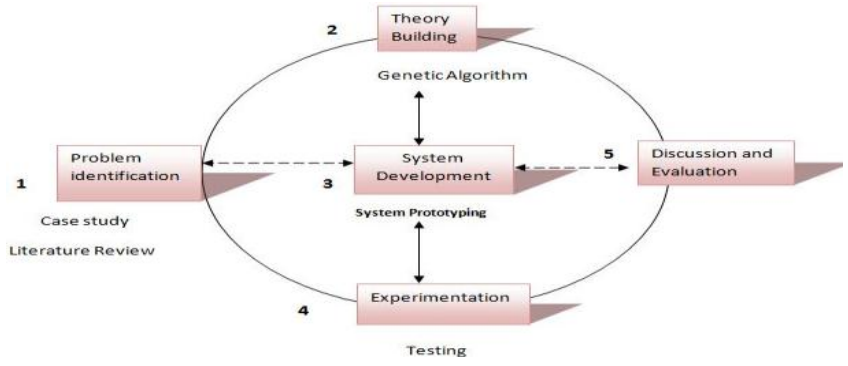

Fig 1: Methodology approach for class timetable

In the real practice, the methodology shown in Figure 1 is an iterative approach across of phases. The strength of this methodology is the ability to track the changes through the process. There are four main elements which are considered during the design of timetable for Sohar University:
1. Lecturer
2. Subject
3. Classroom/lab
4. Time-slot

The following information is considered into the timetable for each semester:

- $\quad$ Subject name with its credit hours and belong to which levels and faculty.

- $\quad$ Flags indicated it's compulsory or pre-request, subject to another subject.

- Consider about available time -slot and the room availability with its capacity, also mention that the class used to be as classroom or labs.

- $\quad$ Each time -slot associated with its day and time.

The Sohar University timetabling involves decision making, and achieving the overall objectives based on a set of constraints, and available resources. Thus, a feasible solution must group all the lectures, classes, and rooms together to produce the timetable that fulfills a set of constraints.

Generally, there are two types of constraints: Hard and soft constraints. These constraints need to produce optimal solutions. Hard constraint is a constraint that is inviolable (rigidly enforce) and must be satisfied in to create a feasible solution. On the other hand, soft constraints are those that are desirable but not essential. It is usually impossible to satisfy all soft constraints. Thus, the acceptance of a feasible solution depends on the degree to which soft constraints are needed to be satisfied. The hard constraints for University timetabling are identified as follows:

1. No student has more than one class at the same time

$$
\begin{aligned}
\text { i. } \quad \forall(i, j), i \neq j, \Delta\left(S_{i} \neq, S_{j} \Delta T S_{i} \neq\right. \\
\left.\quad T S_{j}, \text { where } R_{i,} \neq R_{j,}\right)
\end{aligned}
$$

2. No lecturer can teach more than one class at the same times.

3. No lecturer can be in two classes as the same period. 
i. $\forall(i, j), i \neq j, \Delta\left(L_{i} \neq, L_{j}, \Delta T S_{i} \neq T S_{j}\right)$

4. Consider room availability and the number of students register for the subject:

i. $\forall i$, studentregsiter $\left(S_{i}\right) \leq$ capacity $\left(R_{i}\right)$, where studentregister $(y) \in$ $i, y \in i$, and $\operatorname{Capacity}(y) \in i, y \in R$

Besides that, the soft constrain for the University identified as follows:

1. The room should be suitable for the requirement needs.

2. Each class and lecturer must be presented in a predefined number of hours in the timetable. (Number of hours, assign for each lecturer by the Head of the department and the coordinator of the subject).In addition to that, the teaching load of the lecture does not exceed the maximum load which decided by the managers of Sohar University.

$$
\begin{aligned}
\forall a, \operatorname{load}(a)=\sum_{k=1}^{m}\left(L_{k}, a\right), a \in L, \operatorname{Load}(y) \\
\in i, y \in L
\end{aligned}
$$

The fitness value will be from 0 to 1 , so the evaluation function can be presented as follows to calculate the fitness value:

$$
\operatorname{sum}(x) * 0.2 /(\text { number of subject } * \text { Day) }
$$

Theory Building includes the development of methods or models. GA was investigated and relevant literature was examined in order to determine an effective scheme which represents timetables as chromosomes. Chromosomes are typically represented as simple strings of data and instructions. In this case, chromosomes chose to represent a solution and subjects had been chosen as a gene. Figure 2 explains the framework for the timetabling problem .It can be implemented for solving timetabling problem in any educational institutes, and consider about the constraint of the institute.

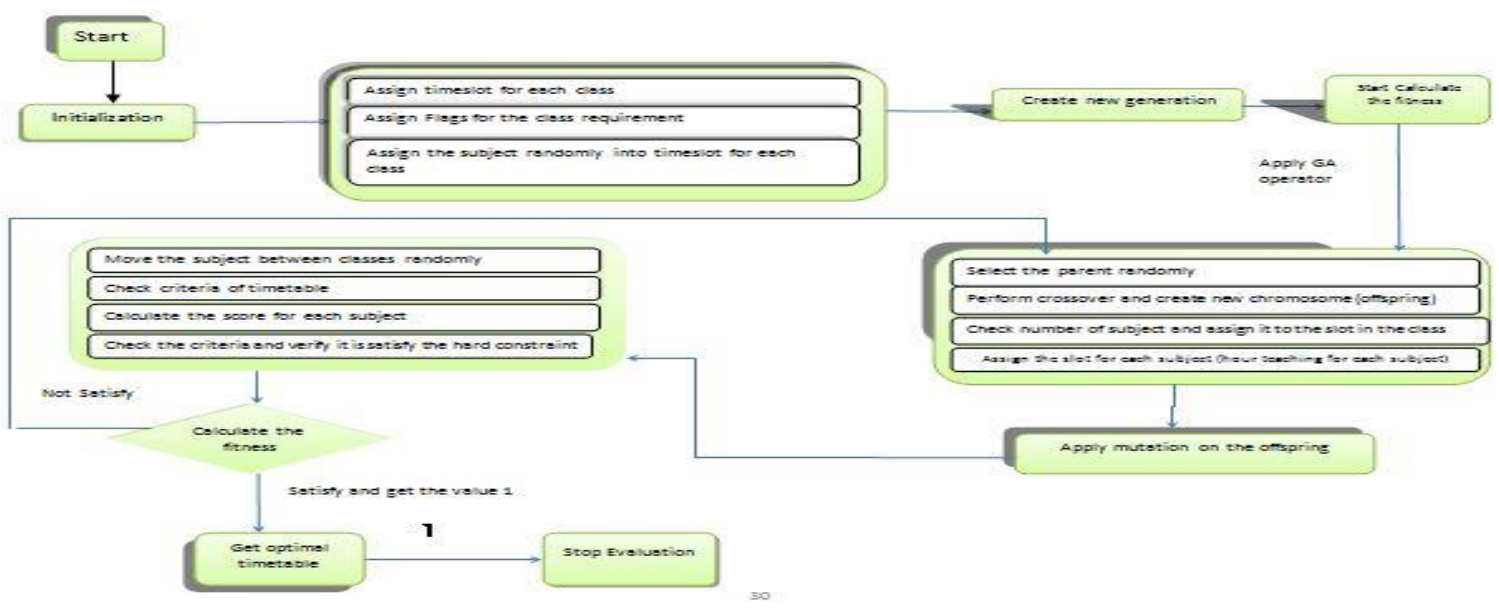

Fig 2: Detailed of GA for the timetabling framework

\section{FINDING AND RESULTS}

The system prototype runs many times using different parameters. The number of time slots was fixed at 40 equal time-slots for 5 days with different number of rooms. Each subject allocated to a time slot ranges between 8 am to $4 \mathrm{pm}$. The default parameters set are shown in Table 1

Table 1 Default parameters setting

\begin{tabular}{|l|l|}
\hline Parameters setting & Variable \\
\hline Slot Number & 40 for each class \\
\hline Population Size & 2500 \\
\hline Elitism & Yes \\
\hline Type of Crossover & Two Point \\
\hline Probability of crossover & $100 \%$ \\
\hline Probability of Mutation & $100 \%$ \\
\hline
\end{tabular}

The best generation of 13 classrooms with 27 subjects is represented by the fitness value equal to 1 that is shown in figure 3 . According to figure 3 there are no clashes between the chromosomes and satisfied all the hard constraints.

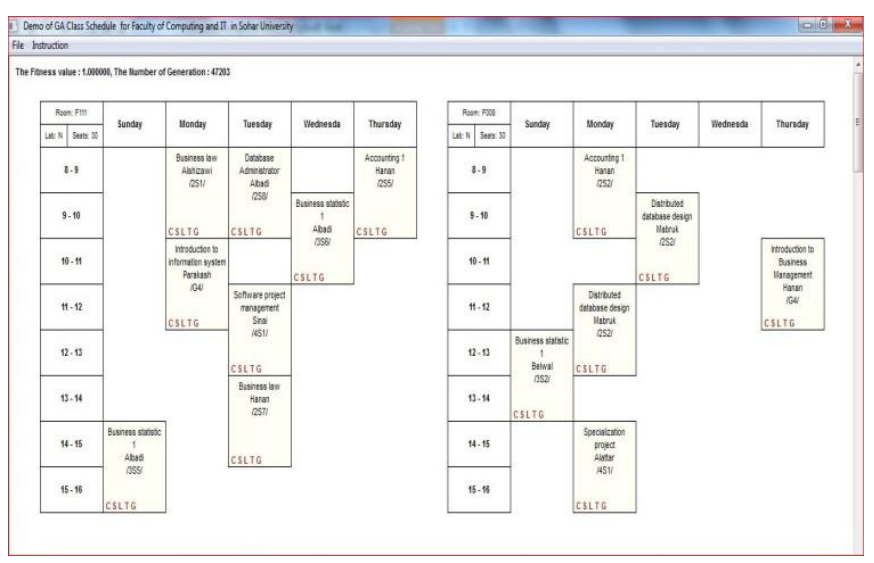

Fig 3: An acceptable timetable. 
The above Figure illustrates the fitness value $=1$ and the number of generations is 47203. However, the worst generations of two classrooms that do not satisfy the hard constraint illustrate in figure 4

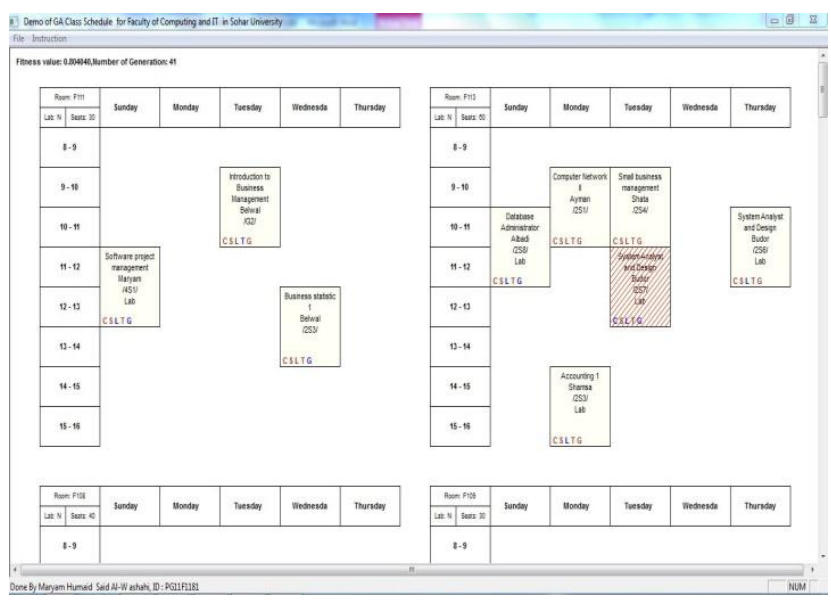

Fig 4. Hard constraint that is not satisfied

Experiments have been done with a several testing using a different set of parameters. Combination of crossover rate and mutation rate should be obtained with an optimal solution. For the first testing, crossover rate is increased and decreased from the default setting. Meanwhile, mutation rate has been applied with different rates. The Table 2 , show the different generation for the fitness value equal to one, that display the optimal solution for the FCIT of Sohar university with different time and different probability of crossover and mutation.

Table 2: The results from Test 1 to Test 12 sequentially

\begin{tabular}{|c|c|c|c|c|}
\hline $\begin{array}{l}\text { Number of } \\
\text { chromosom } \\
\text { es }\end{array}$ & $\begin{array}{l}\text { Crossover } \\
\text { probabilit } \\
\mathrm{y}\end{array}$ & $\begin{array}{l}\text { Mutation } \\
\text { probabili } \\
\text { ty }\end{array}$ & $\begin{array}{l}\text { Time } \\
\text { consumes } \\
\text { (MINUATES } \\
\text { ) }\end{array}$ & $\begin{array}{l}\text { Number } \\
\text { of } \\
\text { generation } \\
\mathrm{s}\end{array}$ \\
\hline 10 & $100 \%$ & $100 \%$ & 13 & 7793 \\
\hline 10 & $100 \%$ & $1 \%$ & 48 & 13434 \\
\hline 10 & $50 \%$ & $100 \%$ & 14 & 7642 \\
\hline 10 & $50 \%$ & $100 \%$ & 20 & 11502 \\
\hline 10 & $50 \%$ & $50 \%$ & 18 & 9480 \\
\hline 10 & $20 \%$ & $30 \%$ & 39 & 13388 \\
\hline 10 & $30 \%$ & $30 \%$ & 26 & 10856 \\
\hline 4 & $100 \%$ & $100 \%$ & 20 & 13984 \\
\hline 4 & $100 \%$ & $1 \%$ & 60 & 17400 \\
\hline 8 & $25 \%$ & $100 \%$ & 23 & 6808 \\
\hline 8 & $100 \%$ & $25 \%$ & 40 & 9644 \\
\hline 8 & $95 \%$ & $95 \%$ & 35 & 9892 \\
\hline
\end{tabular}

According to table 2 show that test number one takes less amount of time to produce a timetable for 27 subjects with 13 rooms without any overlapping of classes, lecturer or student group. However, the result shows different time of generating the timetable from one test to another. The average of generating the best value for one faculty may take 23 minutes.

The following testing in table 3 was conducted using different population size with $100 \%$ crossover rate and $90 \%$ mutation probability. The below table illustrate the result obtained from the experimentation. The different population size shows good result in different testing.

Table 3: Results from Test 40 to Test 43

\begin{tabular}{|l|l|l|l|l|}
\hline $\begin{array}{l}\text { Populatio } \\
\mathrm{n} \text { size }\end{array}$ & $\begin{array}{l}\text { Crossover } \\
\text { probabilit } \\
\mathrm{y}\end{array}$ & $\begin{array}{l}\text { Mutation } \\
\text { probabilit } \\
\mathrm{y}\end{array}$ & $\begin{array}{l}\text { Approximat } \\
\mathrm{e} \\
\text { time(Minute } \\
\text { s) }\end{array}$ & Generation \\
\hline 1500 & 100 & 90 & 25 & 7304 \\
\hline 1000 & 100 & 90 & 46 & 12176 \\
\hline 50 & 100 & 90 & 30 & 7304 \\
\hline 500 & 100 & 90 & 45 & 12850 \\
\hline
\end{tabular}

The prototype of a class timetable is developed effectively. The prototype can be used before beginning of new semester start. The proposed prototype is requiring the least amount of time compared with manual way and produce different acceptable timetable. It assists the Sohar University to get optimal timetable to satisfy both hard and soft constraint.

\section{CONCLUSION AND FUTURE RESEARCH DIRECTIONS}

The GA techniques are useful for solving scheduling problem such as timetable. GA is one of the techniques used to produce the effective and optimized solution. The methodology proposed to be followed in the Sohar University is consisting of the problem identification which explains the constraint and the criteria of timetable based on the university policy. An algorithm has been developed and implemented. Various testing has been produced using different parameters of population size, crossover and mutation probability to find an optimal solution. The result shows the rate of crossover and mutation equal to 100 performed best optimal solutions. The bigger generation also can be as better results. However, changing different number of population size also, it effects in the time length of generating the timetable. Further improvement can be achieved by enhancing the fitness function and used selection mechanism to the algorithm.

\section{REFERENCES}

[1] Schaerf , A., "A Survey of Automated Timetabling", Artificial Intelligence review, 1999, [Online] Available http://www.diegm.uniud.it/satt/papers/Scha99.pdf

[2] Sigl Branimir, Marin Golub, Vedran Mornar, "Solving Timetable Scheduling Problem by Using Genetic Algorithms",

[3] Yang and Petrovic, "A Novel Similarity Measure for Heuristic Selection in Examination", PATAT 2004 Proceedings of the 5th International Conference on the Practice and Theory of Automated Timetabling.

[4] Sandhu Kuldeep Singh, "Automating Class Schedule Generation in the context of a University Timetabling Information System", Thesis submitted to School of Management, Nathan campus, Griffith University, 2001. 
[5] Ehrgott M and Gandibleux X, “ A Survey and Annotated Bibliography of Multiobjective Combinatorial Optimization”, OR Spectrum, 2004.

[6] B. Szpigel, "Optimal train scheduling on a single track railway", Proceedings of IFORS Conference on Operational Research'72, 343-352.

[7] R.Sivaraj, "A REVIEW OF SELECTION METHODS IN GENETIC ALGORITHMS”, International Journal of Engineering Science and Technology(IJEST), vol. 3, MAY 2001, p. 3792.

[8] José-Revuelta, L.M.S.,"A Hybrid GA-TS Technique with Dynamic Operators and its Application to Channel Equalization and Fiber Tracking", [Online], Available: http://cdn.intechweb.org/pdfs/4594.pdf $[01$ September 2013.

[9] K. F. Man, K. S. Tang, and S. Kwong, " Genetic Algorithms: Concepts and Applications", IEEE TRANSACTIONS ON INDUSTRIAL ELECTRONICS, VOL. 43, NO. 5, OCTOBER 1996.
[10] Ehsan Alirezaei, Z.V.a.M.G.-G., "Parallel Hybrid Meta Heuristic Algorithm for University Course Timetabling Problem (PHACT)", '20th Iranian Conference on Electrical Engineering, (ICEE2012)'. held May 1517,2012 at Tehran.Iran: IEEE,673-678.

[11] Chinnasri, W., Krootjohn, S. ,Sureerattanan, N. , "Performance comparison of Genetic Algorithm's crossover operators on University Course Timetabling Problem",'In anon .ED.Computing Technology and Information Management (ICCM), 2012 8th International Conference ' held 24-26 April 2012 at Seoul, Korea (South) . King Mongkut"s Univ. Of Technol: IEEE, 781-786.

[12] Lukas, S., Aribowo, A., Muchri, M.,"Genetic algorithm and heuristic search for solving timetable problem case study: Universities Pelita Harapan timetable", Applications of Digital Information and Web Technologies, 2009. ICADIWT '09. Second International Conference on the , vol., no., pp.629,633, 4-6 Aug. 2009 doi: 10.1109/ICADIWT.2009.5273979 